\title{
SCREENING METHODS OF COXOFEMURAL DYSPLASIA IN DOGS
}

\author{
Antonina Dumitriu, Valeriu Enciu
}

\begin{abstract}
Agrarian State University of Moldova, Chisinau, Republic of Moldova e-mail: toniadumitriu@gmail.com
\end{abstract}

\begin{abstract}
The major desideratum of the screening action of the hip dysplasia is the early discovery of the disease and its highlighting at an early stage. Hip dysplasia is interpreted as an abnormal and / or defective development of the hip (hip joint), which can cause excessive wear of the articular cartilage due to the support of body weight leading to the development of arthritis, often called degenerative joint disease or simply osteoarthritis, these terms being used randomly. All these morphofunctional changes can be detected early, by different methods of observation, which necessarily involve X-ray radiological examination, performing a complex analysis of the images studied, according to the recommendations of various international organizations such as FCI Fédération Cynologique Internationale (FCI) - score which includes measuring the Norberg angle, the Orthopedic Foundation for Animals (OFA), the PennHIP Program - which involves measuring hip laxity, etc.
\end{abstract}

\section{Introduction}

Coxo-femoral dysplasia is a disease characteristic of dogs, categorized as hereditary, which can be transmitted to offspring within 14 generations [18]. This causes morphological changes in the coxo-femoral joint [1], which is manifested by wiping the acetabular cavity in different degrees, flattening the articular head of the femur, increasing the intra-articular distance [2], and functionally, evolving from lack of clinical signs to severe lameness, without a direct correlation with the severity of morphological lesions. Affected joints usually develop varying degrees of synovial inflammation, joint cartilage damage, osteophytes and sclerosis, and subchondral bone remodeling [2]. Also common changes characteristic of hip dysplasia are associated with environmental factors, such as nutrition [10], exercise [8], and skeletal ossification [12].

Canine hip dysplasia was first described in 1935-1937 by Dr. Gerry B. Schnelle, who called it "bilateral congenital subluxation of the hip joint" [14], considering it a rare but rare pathology which currently turns out to be the most common orthopedic condition in dogs. Examining a large number of dogs, he finds that joint deformities have different aspects from one animal to another, which determines the different forms of the disease, depending on the radiological severity of the lesions, in five degrees [15].

In 1966, Henricson, Norberg and Olsson refined the definition of coxofemoral dysplasia, describing it as follows "A different degree of laxity of the hip joint that allows subluxation during early life, giving rise to varying degrees of superficial acetabulum and flattening of the femoral head, eventually inevitably leading to osteoarthritis" [9].

The intensive breeding of purebred dogs makes coxo-femoral dysplasia one of the most studied diseases in this species, and the most intensely studied breed is the German Shepherd, due to its wide range [17].

Subsequently, the disease was diagnosed in almost all races, especially those of large size 
and robust constitution, in which the growth rate at a young age is very high. The most commonly affected are the Terra-nova, Saint-Bernard, Rottweiler, Kuvasz, Giant Schnauzer and Boxer [6], but the disease can also be found in some small breeds. However, there are breeds in which the disease has not been diagnosed, among them we list: Dwarf Pinscher, Borzoi, Greyhound, Bedlington-Terrier, Irish-Terrier, Lakeland-Terrier.

The disease affects both males and females, with coxo-femoral dysplasia being more important in service dogs because they cannot be subjected to prolonged exertion during training, they get tired in a short time and have severe joint pain. For this reason, the disease should be detected as early as possible before dogs are selected for training.

Today, the veterinary consensus is that hip dysplasia is a congenital condition manifested as joint laxity that leads to the development of osteoarthritis.

The aim of the paper was to visually familiarize with the measurement technique on an $\mathrm{X}$-ray to diagnose and assess the degree of hip dysplasia in dogs, performing measurements, using the technique described by Norberg, and assessing the laxity index.

\section{Materials and methods}

The materials were radiographs of 2 dogs, who were diagnosed with X-rays based on images of the pelvis, with the capture of the knee joints. The examined subjects were placed on their backs in a supine position, with the hind limbs extended and parallel to each other, the knee joints being rotated internally with the symmetrical pelvis. The radiography was obtained in direct ventro-dorsal projection, using an ARIA veterinary digital X-ray device at the "Iasi Veterinary Imaging Center”. The examined animals were anesthetized with SEDAM (acepromazine) in combination with VETASED (ketamine) according to the instructions.

The measurements were performed according to the protocols published on the websites: Animal Orthopedic Foundation (OFA) [16], Federation Cynologique Internationale (FCI) [6], Pennsylvania Hip Improvement Program (PennHIP) [13] - where the most exposed are widespread and detailed screening approaches for canine hip dysplasia (CHD).

\section{Results and discussions}

The study methods, imposed by various international organizations, were transposed on two radiographs submitted by colleagues from Iasi. Thus, we performed measurements of the Norberg angle [3] and evaluated the hip laxity - Pennhip [13]. Both techniques require mandatory sedation of dogs until muscle relaxation is achieved. The examination of an radiographic image for the diagnosis and assessment of the degree of hip dysplasia takes into account the acetabular cavity, the femoral head and the secondary changes. Of interest are 9 detailed features: Norberg angle, subluxation, cranial acetabular margin, dorsal acetabular margin, cranial acetabular lip, acetabular fossa, caudal acetabular margin, exostoses of the femoral head and neck and femoral head remodeling.

Theoretically, the Norberg method [3] consists in measuring the angle obtained between a line joining the geometric centers of the two femoral ends and a line starting from the center 
of the femoral head and passing tangentially to the anterior edge of the acetabular cavity. The technique is possible only on radiographic film of sufficient quality and obtained on the animal positioned in supine position with the limbs in forced extension, as far as possible to ensure a parallelism between the femoral bone rays, while achieving a slight internal rotation of the two

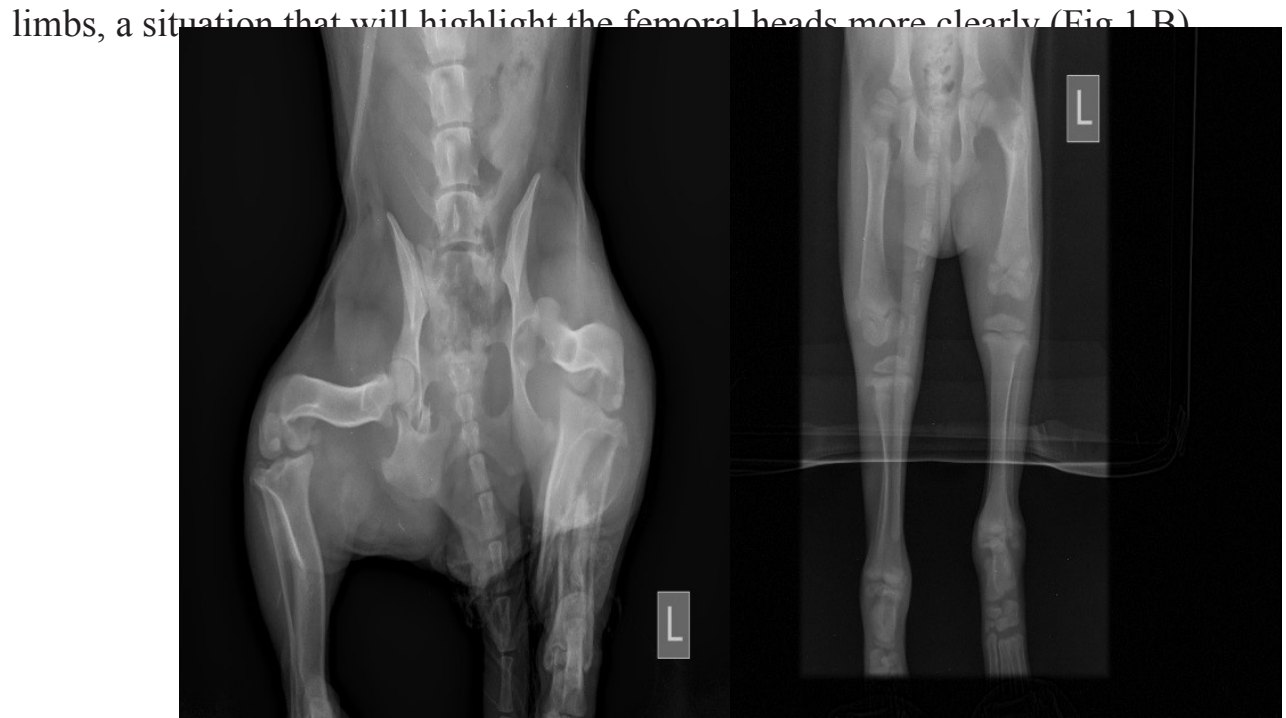

Figure 1. A - dislocation of the hip joint, B - dysplastic joint

The classification by degrees of dysplasia is made both after observing the osteoarticular structural changes and the value of the Norberg angle. 5 degrees of dysplasia are unanimously accepted, as follows:

Grade A (1) -lack of dysplasia. Signs of hip dysplasia are missing, femoral head and acetabulum are congruent. The craniolateral edges have clear contours and rounded ends. The joint space is narrow and smooth. The acetabular angle according to Norberg is approx. 1050 (as a reference). In the case of excellent hip joints, the craniolateral acetabular edge surrounds the femoral head somewhat more distant than laterocaudal.

Grade B (2) - approximately normal joint. The coxo-femoral joint is almost normal, the femoral head and acetabulum are slightly incongruent, the Norberg angle is about 1050, or the center of the femoral head is in a medial position relative to the dorsal edge of the acetabulum, the femoral head and acetabulum are congruent.

Grade C (3) - mild dysplasia. The femoral head and acetabulum are incongruent, the Norberg acetabular angle is approximately 1000 and / or the craniolateral edge of the acetabulum is slightly flattened. There may be irregularities or just fine signs of osteoarticular alterations of the cranial, caudal or dorsal acetabular margin or on the femoral head and neck.

Grade D (4) - moderate dysplasia. Obvious incongruity between the femoral head and the subluxated acetabulum. Acetabular angle according to Norberg greater than 900 (reference only). Flattening of the craniolateral margins and / or signs of osteoarthritis.

Grade E (5) - severe dysplasia. Marked dysplastic changes of the hip joints, such as disloca- 
tions or subluxations [5] (Fig. 1 A), Norberg's right acetabular angle less than 900, obvious flattening of the cranial acetabular margin, deformation of the femoral head (mushroom-shaped, flattened), or other signs of osteoarthritis.

The PennHip method [13] evaluates both the qualitative aspect and the quantitative aspect of the hip by measuring the degree of joint laxity. Joint laxity of the hip is the most important risk factor in the development of osteoarthritis as such it is very important to understand the difference between passive and functional laxity. Functional laxity is the pathological laxity that occurs during the normal season in the dysplastic dog. Passive laxity is the laxity determined by the PennHip method under sedation by measuring the degree of "extension".

The index method is calculated by digitally superimposing precision circle indicators on the cortical edges of the acetabulum and the femoral heads to identify the respective geometric centers. In the compression picture (Fig. 2. A), if the joint does not show osteoarthritis, the centers of the acetabulum and the femoral head should coincide, indicating that the joint is indeed concentric.

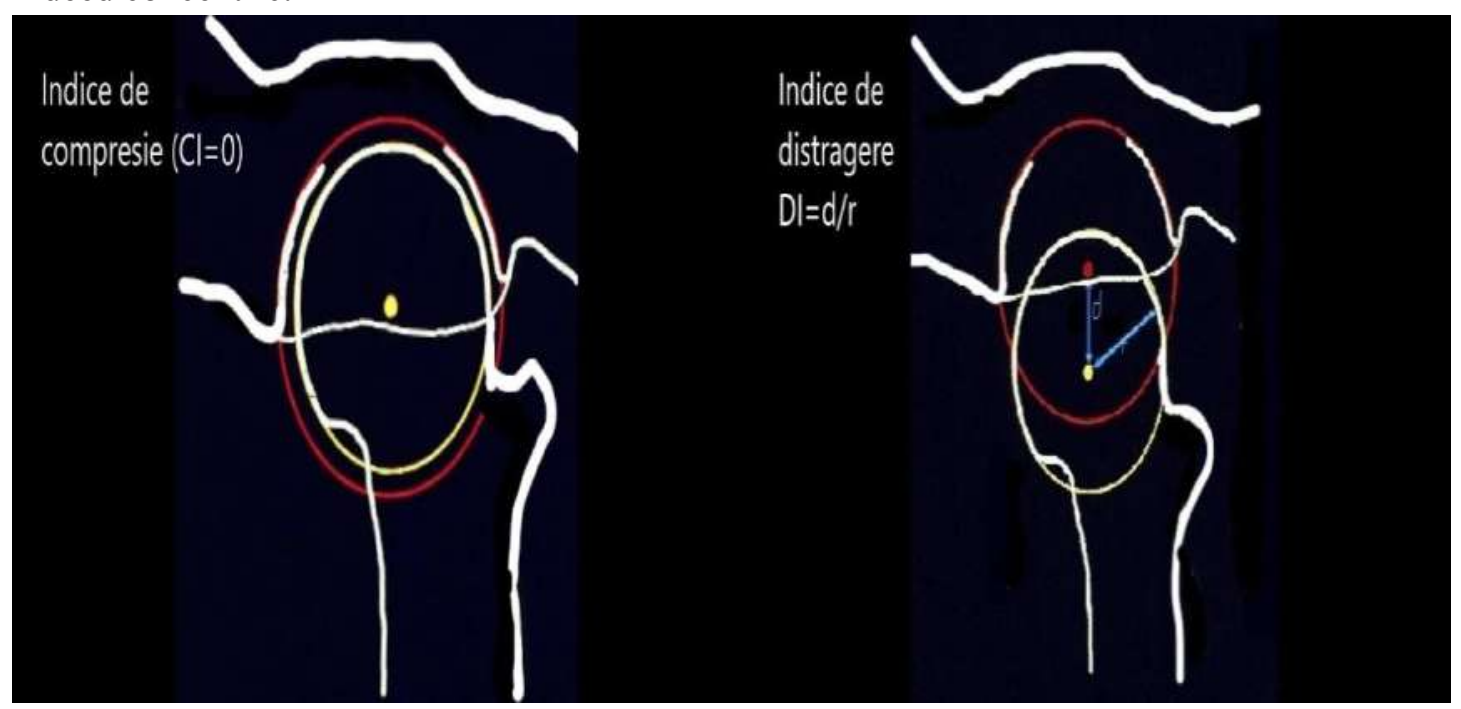

Figure 2. Graphical representation of the compression index (A) and distraction index (B)

From the point of view of distraction (Fig. 2. B) [17], the distracting force determines the separation between the centers. The distance " $\mathrm{d}$ " between the centers is a measure of the laxity of the hip joint. However, "d" also varies depending on the size of the dogs (larger dogs would probably have a larger " $d$ " than smaller dogs). To circumvent these potential sources of variation, "d" is normalized to the size of the femoral head and acetabulum, dividing it by the radius of the femoral head - " $r$ ". The resulting index, Distraction Index $=\mathrm{d} / \mathrm{r}$, is a number without units between 0 and 1 (or more). The laxity index calculated for the compression view is called the compression index $(\mathrm{CI})$, and the laxity index for the distraction view is called the distraction index (DI). Hips with distraction vision DI that are less than 0.3 are considered to be tight, while DIs close to 1 is considered to be extremely weak. DI is an indication of the "percentage of the joint" that the femoral head is displaced from the acetabulum. Therefore, laxity caused 
by DI is called passive hip laxity, as opposed to functional hip laxity, which is the pathological form of hip laxity that occurs in dysplastic hips during weight bearing [17].

Recently, the PennHIP stress index and OA scores have been found to have strong correlations with modified articular cartilage microstructures, potentially indicating a relationship between joint laxity measured by this technique and joint surface degeneration [11].

Table 1 categorizes the degrees of hip dysplasia in dogs depending on the requirements and standards of different international resource organizations.

Table 1. Degrees of coxofemoral dysplasia in dogs

\begin{tabular}{l|c|l|l} 
OFA (USA) & FCI (Europa) & BVA (UK/Australia) & SV (Germania) \\
\hline Excellent & A- 1 & $0-4$ & Normal \\
\hline Good & A- 2 & $5-10$ & Normal \\
\hline Fair & B- 1 & $11-18$ & Normal \\
\hline Borderline & B -2 & $19-25$ & Fast Normal \\
\hline Mild & C & $26-35$ & Noch Zugelasser \\
\hline Moderate & D & $36-50$ & Mittlere \\
\hline Severe & E & $51-106$ & Schwere
\end{tabular}

The minimum age for performing the hip dysplasia test by the PennHip method [13] is 16 weeks and the forced extension method can be applied from the age of 5 months. In both cases it is recommended to repeat the radiographs to stage the changes in case of a positive diagnosis and for certification on the OFA system [16] it is accepted over the age of 24 months and on the British system [4,7] the minimum age should be 12 months.

\section{Conclusions}

Canine hip dysplasia affects millions of canine specimens each year and can result in debilitating orthopedic hip pathology. Many dogs suffer from osteoarthritis, pain and lameness causing material damage to owners through treatments, shortening the life of working dogs and thus reduced performance. The incidence of the disease is very well documented in terms of large breeds of dogs, but it can also occur in small dogs and toys and also in cats. Hip dysplasia is a complex congenital disease of a polygenic nature. Both veterinarians and breeders have tried to eliminate this condition through selective mating strategies, but still the frequency of reduction to purebreds is disappointing.

\section{Bibliography}

1. Barr ARS, Benny HR, Gibbs C. Clinical hip dysplasia in growing dogs: the long-term results of conservative management. J Small Anim Pract. 1987;28:243-252.

2. Bijlsma JW, Berenbaum F, Lafeber FP. Osteoarthritis: an update with relevance for clinical practice. Lancet. 2011;377:2115-2126.

3. Comhaire FH, Criel ACC, Dassy CAA, Guévar PGJ, Snaps FR. Precision, reproducibility, and clinical use fulness of measuring the Norberg angle by means of computerized image analysis. Am J Vet Res. 2009;70:228-235.

4. Dennis R. Interpretation and use of BVA/KC hip scores in dogs. In Practice. 2012;34:178-194. 
5. Farese JP, Todhunter RJ, Lust G, Williams AJ, Dykes NL. Dorsolateral subluxation of hip joints in dogs measured in a weight-bearing position with radiography and computed tomography. Vet Surg. 1998;27: 393-405.

6. Fédération Cynologique Internationale [webpage]. FCI Scientific Commission. http://www.fci. be/en/FCI-Scientific-Commission-71.html, Accessed May-June 2021.

7. Gibbs $\mathrm{C}$. The BVA/KC scoring scheme for control of hip dysplasia: interpretation of criteria. Vet Rec. 1997;141:275-284.

8. Greene LM, Marcellin-Little DJ, Lascelles BD. Associations among exercise duration, lameness severity, and hip joint range of motion in Labrador retriever swith hip dysplasia. J Am Vet Med Assoc. 2013;242: 1528-1533.

9. Henricson, B., Norberg, I., Olsson, SE. :On the etiology and pathogenesis of hip dysplasia: a comparative review. J Small Anim Pract 7:673-88, 1966.

10. Kealy RD, Lawler DF, Ballam JM, et al. Evaluation of the effect of limited food consumption on radiographic evidence of osteoarthritis in dogs. J Am Vet Med Assoc. 2000;217:1678-1680.

11. Lopez MJ, Lewis BP, Swaab ME, Markel MD. Relations hips among measurements obtained by use of computed tomography and radiography and scores of cartilage and microdamage in hip joints with moderate to severe joint laxity of adult dogs. Am J VetRes. 2008;69:362-370.

12. Madsen JS, Reimann I, Svalastoga E. Delayed ossification of the femoral head in dogs with hipdysplasia. J Small Anim Pract. 1991;32: 351-354.

13. PennHIP [webpage]. PennHIP Method: Measuring Hip Joint Laxity. https://antechimagingservices.com/antechweb/measuring-hip-joint-laxity. Accessed May, 2021.

14. Schnelle GB. Bilateral congenital subluxation of the coxo-femoral joints in a dog. University of Pennsylvania Bulletin School of Veterinary Medicine Veterinary Extension Quarterly. 1937;37:15-16.

15. Schnelle, G.B.: The veterinary radiologist: regional radiography of the pelvic region, Part 1 North American Veterinarian 18:53-56, 1937.

16. The Orthopedic Foundation for Animals [webpage]. The OFA's Hip Radiograph Procedures. https://www.ofa.org/diseases/hip-dysplasia. Accessed May-June 2021.

17. Tikekar, A., Soo, M., Lopez-Villalobos, N., Worth, A.J. : Provisional heritability estimates of four distraction index traits in a breeding population of German Shepherd dogs. NZVetJ : 1-16,2018. Pubmed reference: 30122125. DOI: 10.1080/00480169.2018.1512429

18. Филиппов Ю. И., Митин В. Н. Дисплазия тазобедренных суставов у собак, Ветеинария N. 9,1990 , c. $66-69$. 